\title{
Neuordnung der Aufgabenverteilung im Gesundheitswesen Treffen der Entscheidungsträger unter der Leitung der AfnP
}

Die Neuordnung der Aufgabenverteilung beschäftigt im Gesundheitswesen zurzeit verschiedene Ebenen und Berufsgruppen. Grund genug für die AfnP, diese Thematik im Fachbereich Nephrologie aufzugreifen. Am 15.1.2009 lud die AfnP nach Stuttgart ein und versuchte dabei möglichst alle in der Nephrologie relevanten Gruppen und Entscheidungsträger an einen Tisch zu bekommen.

Eingeladen waren die Deutsche Gesellschaft für Nephrologie (DGfN), die Deutsche Arbeitsgemeinschaft Klinische Nephrologie (DAGKN), der Verband Deutscher Nierenzentren (DDnÄ), der Arbeitskreis Saar Pfalz Mosel, der Sachverständigenrat, der Gesundheitsrat Südwest, die Deutsche Krankenhausgesellschaft e. V. (DKG), das Kuratorium für Dialyse und Nierentransplantation e. V. (KfH), die Patienten Heimversorgung (PHV), das Institut der Fort und Weiterbildung der PHV (ifw), die Bundesarbeitsgemeinschaft der Nephrologischen Weiterbildungsstätten, die Vertretungen aus den Weiterbildungsstätten Nürnberg, Hamburg, Traunstein, Ulm, der Arbeitskreis Transplantationspflege e. V. (AKTX) und der Fachverband nephrologische Berufsgruppen (fnb).

\section{Fachkräftemangel im ärztlichen und pflegerischen Bereich}

Ralf Neiheiser von der DKG sprach über die demografische Entwicklung und den daraus zwangsläufig entstehenden Fachkräftemangel sowohl im ärztlichen als auch im pflegerischen Bereich. Der deutsche Pflegegipfel, der im September 2008 in Berlin stattfand, hatte eine Arbeitsgruppe zu dieser Thematik gebildet, die am 6.2.2009 zum 1. Mal tagte. Die Studie des DKI (Deutsches Krankenhaus Institut e. V.) mit dem Titel „Neuordnung von Aufgaben des ärztlichen Dienstes“ ist auf der Internetseite der DKG nachzulesen. Dort findet sich auch die Zeitschrift „Krankenhaus Barometer", die sich mit der Thematik befasst. Neiheiser stellte verschiedene neue Fördermöglichkeiten vor, unter anderem das Förderprogramm zur Neueinstellung von Pflegkräften: das Krankenhausfinanzierungsreformgesetz (KHRG). Im Verlauf wurde diskutiert, ob und wie die einzelnen Berufsgruppen der Pflege in der Nephrologie eingesetzt werden können. Zur Sprache kamen hier Altenpfleger, ebenso wie die jetzt schon zahlreich in der Dialyse tätigen medizinischen Fachangestellten und Absolventen von Pflegestudiengängen.

\section{Der rechtliche Rahmen muss} erheblich angepasst werden

Prof. Hans Kamps beleuchtete als Jurist das Thema aus rechtlicher Sicht: Eine Neuordnung der Aufgabenverteilung muss derzeit in Deutschland scheitern, da die Ausübung ärztlicher Tätigkeiten in mehreren Gesetzen klar geregelt ist (§ 2 BÄO, $§ 1, \S 5$ HeilprakG, $\S 613, \S 278$ BGB). Bevor also eine Neuregelung kommen kann, müsste vorher eine gesetzliche Grundlage geschaffen werden. Hierfür sieht er jedoch weder Möglichkeit noch Notwendigkeit. Wie Kamps betonte, ginge mit der eigenständigen Übernahme ärztlicher Tätigkeiten auch die Verantwort-

Ausbildungsziele laut Krankenpflegegesetz eigenverantwortlich

- eigenständige Grund- und Behandlungspflege

- den Pflegebedarf erheben und feststellen

- Organisation, Durchführung, Dokumentation und Evaluation der Pflege

- beraten, anleiten, unterstützen

- Menschen beim Gesundwerden begleiten

- Patienten beobachten und Bedürfnisse erkennen

- lebenserhaltende Sofortmaßnahmen einleiten

- Bindeglied zwischen Arzt und Patient

- Organisation der Pflegeüberleitung

- Sicherung und Mitwirkung am Qualitätsmanagement

- Anleitung zur Selbsthilfe

- Gesundheitsvorsorge und Rehabilitation

- Pflegehilfsmittel

im Rahmen der Mitwirkung

- eigenständige Durchführung ärztlich veranlasster Maßnahmen

- Maßnahmen der medizinischen Diagnostik, Therapie und Rehabilitation

- Maßnahmen in Krisen- und Katastrophensituationen

- interdisziplinäre Zusammenarbeit

(KrPflG von 2003, Abschnitt 2 / § 3 / Abs. 1) 
lichkeit und somit die Haftung auf das Pflegepersonal über.

Wie Marion Bundschu, Vorsitzende der AfnP und Leitung der nephrologischen Weiterbildungsstätte Ulm, anhand der einzelnen Ausbildungscurricula für die verschiedenen Berufsgruppen in der Nephrologie zeigte, besteht auch hier ein großer Handlungsbedarf. Um die Übernahme ärztlicher Tätigkeiten zu ermöglichen, müsste zunächst die Ausbildung erheblich angepasst werden. Auch das Krankenpflegegesetz (KrPflG) aus dem Jahr 2003 unterscheidet zwischen eigenverantwortlichen Aufgaben der Pflege und Aufgaben im Rahmen von Mitwirkung (siehe Kasten). Es lässt in seiner derzeitigen Fassung nur die eigenständige Durchführung ärztlich veranlasster Maßnahmen, nicht aber die eigenständige Veranlassung dieser Maßnahmen zu (§ 3). Die Ausbildung zum Gesundheitspfleger/Krankenpfleger soll laut KrPfG § 3 vom 1.1.2004

entsprechend dem allgemein anerkannten Stand pflegewissenschaftlicher, medizinischer und weiterer bezugswissenschaftlicher Erkenntnisse fachliche, personale, soziale und methodische Kompetenzen zur verantwortlichen Mitwirkung insbesondere bei der Heilung, Erkennung und Verhütung von Krankheiten vermitteln.

Hier ist allerdings schon eine Änderung in Planung.

Die 2-jährige Fachweiterbildung zur „Fachpflegekraft für Nephrologie“ hat das Ziel, die Krankenschwester mit den vielfältigen Aufgaben in den verschiedenen Bereichen der nephrologischen Pflege vertraut zu machen und ihnen die zur Erfüllung dieser Aufgabe erforderlichen speziellen Kenntnisse, Fertigkeiten, Verhaltensweisen und Einstellungen zu vermitteln. Zu den Aufgaben gehören

- geplante fachkundige Pflege

- Überwachung und Mitwirkung bei der Nierenersatztherapie

- Unterstützung ärztlichen Handelns bei der Durchführung und Überwachung fachspezifischer, therapeutischer und diagnostischer Maßnahmen

- sachgerechter Umgang mit Medizinprodukten und Hilfsmitteln

- Mitwirkung an bzw. gegebenenfalls Einleitung von Notfallmaßnahmen

- Qualitätssicherung
- Anleitung bzw. Beratung von Patienten und Angehörigen

- Einhaltung bzw. Überwachung der Hygiene

- Einhaltung bzw. Überwachung der Unfallvorschriften und Arbeitssicherheit

Der Sachverständigenrat zur Begutachtung der Entwicklung im Gesundheitswesen hat 2007 im Auftrag der Bundesregierung ein Gutachten mit dem Titel „Kooperation und Verantwortung - Voraussetzung einer zielorientierten Gesundheitsversorgung“ erstellt. Dieses Gutachten ist die Basis für das am 1.7.2008 in Kraft getretene Pflegeweiterentwicklungsgesetz (PfWG). Wenn die Voraussetzungen im Bereich der Gesetzgebung und der Ausbildung gegeben sind, könne sich durchaus eine andere Aufgabenverteilung in der Nephrologie ergeben, resümierte Bundschu.

\section{Perspektiven der Aus- und Weiterbildung}

Stefanie Schlieben, Leiterin der Berufsfachschule für Krankenpflegehilfe in München, referierte anschließend über Perspektiven der Aus- und Weiterbildung in den Pflegeberufen. Sie zeigte Wege und Möglichkeiten in der heutigen Pflegeausbildung auf, einschließlich der intensiver werdenden akademischen Ausbildung. Allerdings ist auch hier noch nicht ausreichend geklärt, welche Aufgaben ausschließlich studierte Pflegekräfte am Patienten ausüben dürfen. In der heutigen Krankenpflegeausbildung lege man großen Wert auf die Kompetenzorientierung, was zum Verlust von fachlichen Inhalten führt. Dies ist somit kontraproduktiv zu Bestrebungen, dem Pflegepersonal eine eigenständige Veranlassung von Maßnahmen zu übertragen.

Auch Schlieben sieht die Möglichkeit, bei einer entsprechenden Qualifikation und Klärung der rechtlichen Voraussetzungen eine Neuverteilung von Aufgaben vorzunehmen. Allerdings seien in den letzten 10 Jahren 50000 Stellen im Bereich der Pflege abgebaut worden, weshalb auch hier ein Personalmangel bestehe. Das Verlegen ärztlicher Aufgaben in den Bereich der Pflege verschiebe nur die Probleme, löse sie aber nicht. Einen ausführlichen Artikel hierzu veröffentlichte sie in der Ausgabe 5/2008 von Dialyse aktuell.
Ärzte und Pflegende sollten einen Konsens finden

Im Anschluss an die Vorträge kam es zu einer lebhaften Diskussion. Insbesondere ein Arbeitspapier von einigen Mitgliedern der Bundesarbeitsgemeinschaft nephrologischer Weiterbildungsstätten wurde heftig diskutiert. Wie Dr. Weinreich zu bedenken gab, dürften einige der in diesem Papier aufgeführten Tätigkeiten selbst von Ärzten nur mit einer umfangreichen Zusatzausbildung durchgeführt werden. Solche Tätigkeiten sollten auf keinen Fall den Pflegekräften übertragen werden, da ihnen hierfür die fachliche Basis fehle. Es gebe jedoch heute schon eine Reihe von Tätigkeiten, die delegiert werden könnten, und diese Möglichkeit gelte es auszuschöpfen.

Es gibt noch eine Menge Handlungsbedarf und Ärzte und Pflegende sollten gemeinsam einen Konsens finden - das war Schlusstenor. Alle derzeitigen Ausbildungen müssen in Ihren Ausbildungsinhalten den derzeitigen Bedingungen (Altersstruktur, Zunahme multimorbider Patienten) angepasst werden. Die AfnP wird an dieser Sache mit allen Berufsgruppen aktiv weiterarbeiten. Weitere Zusammenkünfte sind angedacht, bei denen die einzelnen Berufsgruppen auf der Basis der heutigen Diskussion ihre erarbeiteten Vorschläge zur Neuordnung der Aufgabenverteilung in der Nephrologie vorstellen. Vieles ist derzeit im Umbruch und es ist umso wichtiger, dass bei der Neugestaltung im Gesundheitswesen auch gerade die Pflege aktiv mitarbeitet. Sollten Sie zu diesem aktuellen Thema Anregungen haben oder aktiv mitarbeiten wollen, so wenden Sie sich an die AfnP-Geschäftsstelle.

Marion Bundschu, Ulm, und Hans-Martin Schröder, Neuwied

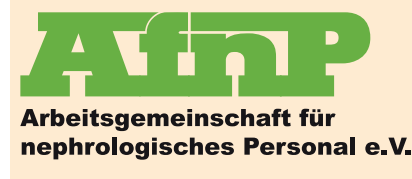

So können Sie uns erreichen: AfnP Geschäftsstelle Käppelesweg 8; 89129 Langenau Tel.: 07345/22933; Fax: 0 7345/7540 E-Mail: info@afnp.de; Internet: www.afnp.de

Vorstand der AfnP e.V.

- Marion Bundschu (1. Vorsitzende)

- Hans-Martin Schröder (stellv. Vorsitzender)

- Gabi Steck (Schatzmeisterin)

- Helga Damaschke (Schriftführerin) 